\title{
DEPARTAMENTO DE CIENCIAS QUÍMICO-BIOLÓGICAS: INICIOS Y PERSPECTIVAS DE ASESORÍA ACADÉMICA
}

\author{
Chemical-Biological Science Department: Beginning and \\ Prospects of Academic Advisory
}

\section{EPISTEMUS}

ISSN: 2007-8196 (electrónico)

ISSN: 2007-4530 (impresa)

María Rosa Estela Lerma Maldonado1

José Manuel Aguilar García ${ }^{2}$

María Alba Guadalupe Corella Madueño 3

Rosa Marina Arvayo Ortiz 4

Julio Cesar Lozano Aviles 5

Recibido: 20 de septiembre de 2016,

Aceptado: 30 de noviembre de 2016

\section{Autor de Correspondencia:}

M. C. María Rosa Estela Lerma Maldonado

Correo: rlerma@guayacan.uson.mx

\section{Resumen}

El "Programa de Asesorías Académicas en el Área Básica del Departamento de Ciencias Químico Biológicas" es un programa continuo que inició en el semestre 2008-2, a petición de los Comités Evaluadores CIEES, como respuesta al bajo porcentaje en aprobación de las asignaturas del área básica y a la alta incidencia de alumnos en riesgo. Las asesorías fueron desarrolladas por maestros de tiempo completo, adscritos al Departamento. Los alumnos fueron convocados a participar voluntariamente. Se muestran los resultados obtenidos en los dos primeros semestres de implementación del programa. En el primer periodo asistieron 58 alumnos, de los cuales aprobaron 44 (75.8\%) y en el segundo asistieron 30, aprobando 22 (73.3\%). Se observa que un alto porcentaje de los alumnos voluntarios del programa, lograron aprobar las asignaturas reincidentes. En el presente estudio se logró definir la problemática y en consecuencia, permitió proponer una estrategia correctiva potencial para solucionarla.

Palabras clave: Asesorías, alumnos, universidad.

\section{Abstract}

The "Academic Advising Program in the Basic Area of the Department of Chemical and Biological Sciences" is a continuous program, which began in the 2008-2 semester at the request of the CIEES in response to the low approval percentage and high incidence of repeat students in the basic area. The advisories were developed by full-time teachers, assigned to the Department. Students were invited to participate voluntarily. The results obtained during the first year that the program was implemented are showed. In the first period attended by 58 students, of which 44 approved (75.8\%) and the second was attended by 30, approving 22 (73.3\%). It is noted that a high percentage of volunteer students in the program, managed to pass the recidivists subjects. In the present study it was possible to define the problem and thus allowed us to propose a potential corrective strategy to solve it.

Keywords: Advisories, student, university
Departamento de Ciencias Químico Biológicas, Universidad de Sonora/Correo: rlerma@guayacan.uson.mx 1 Departamento de Ciencias Químico Biológicas, Universidad de Sonora/Correo: jaguilar@guayacan.uson.mx 2 Departamento de Ciencias Químico Biológicas, Universidad de Sonora/Correo: Icorella@guayacan.uson.mx 3

Departamento de Ciencias Químico Biológicas, Universidad de Sonora/Correo: marar@guayacan.uson.mx 4 


\section{INTRODUCCIÓN}

Ante una sociedad globalizada, cuya dinámica se sustenta esencialmente en el conocimiento y la información, la educación superior mexicana requiere transformar su forma de operación e interacción con la sociedad. Todo programa educativo deberá aspirar a la formación de profesionales que cubran los perfiles más exigentes del medio laboral e intelectual, tanto en los planos técnico, profesional y científico, como en el plano de la formación de la nueva ciudadanía [1]. Para cumplir con esta nueva visión, es necesario establecer un nuevo paradigma, encaminado a mejorar la formación de los estudiantes, mediante el ofrecimiento de una educación de calidad [2].

En este escenario, la atención personalizada al estudiante constituye, sin lugar a dudas, un recurso de gran valor, que implica visualizar al alumno como participante activo del proceso formativo, en lugar de ser un receptor pasivo. Además, esta estrategia propicia el logro del desarrollo integral, contribuye a la adaptación del estudiante al ambiente escolar y al fortalecimiento de sus habilidades de estudio y de trabajo [3]. Entre los elementos más importantes para impulsar esta transformación está la asesoría académica, que se define como un medio de comunicación abierta y libre entre el alumno, que quiere adquirir una preparación profesional y humana, y el profesor, que está comprometido en la búsqueda y enseñanza de la verdad [4]. Este tipo de atención puede ayudar, adicionalmente, a abatir los índices de reprobación y rezago escolares, a disminuir las tasas de deserción y a mejorar el porcentaje de egreso [5].

Otro de los principales problemas de la Educación Superior en México, se refiere a los altos índices de reprobación de materias y la baja eficiencia terminal de los egresados [6], problema que no es reciente; hace ya casi dos décadas, Díaz de Cossío (1998) mencionaba que en la educación superior mexicana, de cada 100 alumnos que ingresaban, 60 terminaban las materias contenidas en el plan de estudios en un lapso de cinco años y de éstos, sólo 20 se titulaban. De los que se titulaban, sólo dos (10\%) lo lograban en edades entre 24 o 25 años; el resto lo hacía en edades entre 27 y 60 años [5].

Nuestra institución no está exenta de esta problemática, puesto que los estudiantes que ingresan a las licenciaturas ofrecidas por el Departamento de Ciencias Químico Biológicas, presentan dificultad para aprobar en los primeros semestres, algunas de las materias del eje básico, principalmente del área de matemáticas, física y química. De hecho, algunos estudiantes se ven en la necesidad de cursar por segunda o incluso por tercera vez alguna materia del eje básico. Esto trae consigo una situación de descontrol, que, en muchas ocasiones, lleva al alumno a un cambio de licenciatura o en casos extremos, a abandonar los estudios universitarios [7].

\section{DESARROLLO}

La jefatura del Departamento de Ciencias Químico Biológicas, tomando en cuenta la sugerencia de los Comités Interinstitucionales para la Evaluación de la Educación Superior (CIEES), solicitó a un grupo de docentes del área

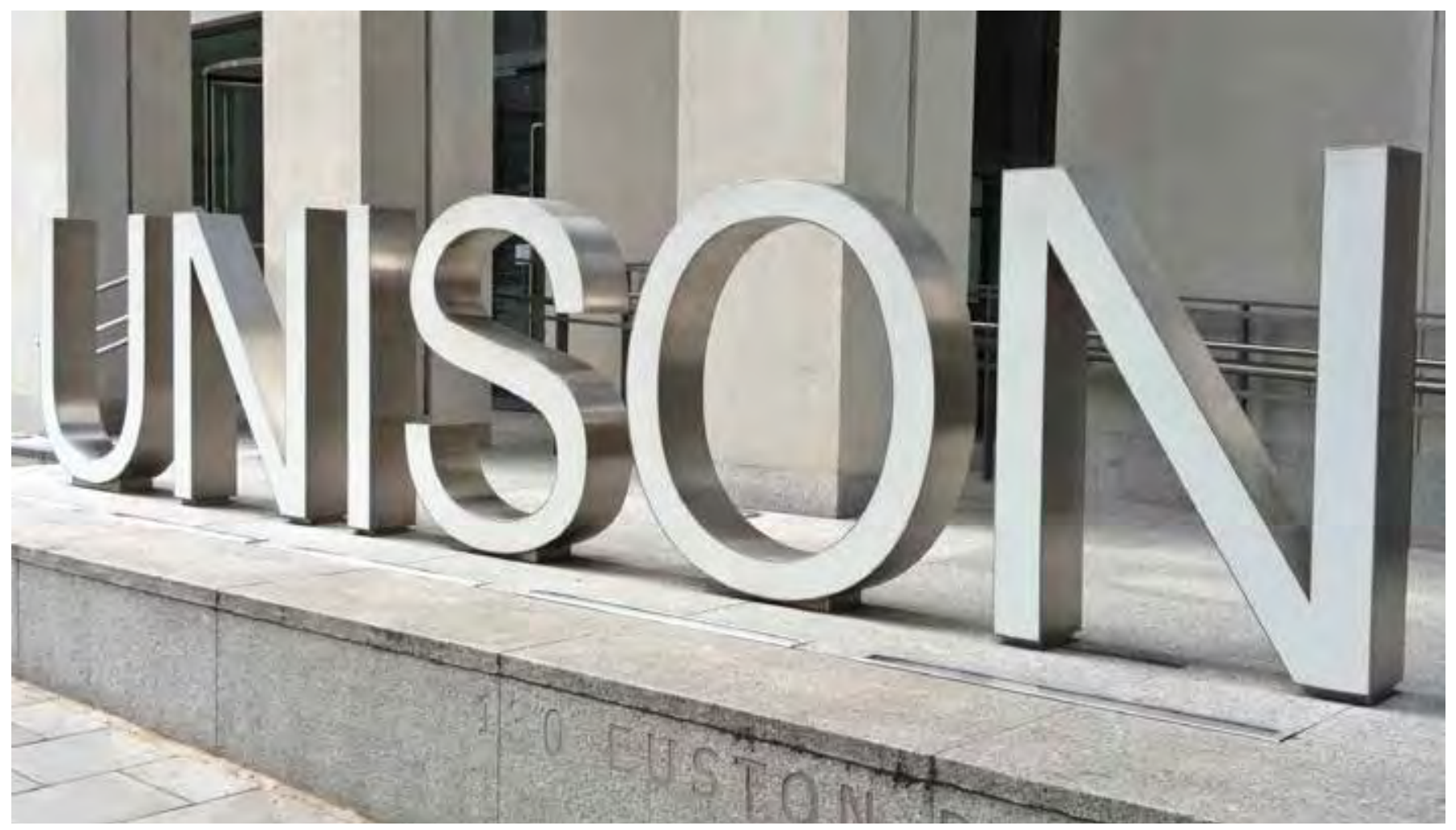


básica, diseñar una estrategia para mejorar el índice de reprobación de las materias de Química del área básica, lo que llevó al planteamiento de un programa con el siguiente objetivo general: Implementar un programa permanente de asesoría académica en las asignaturas de Química del área básica del Departamento de Ciencias Químico Biológicas, en cumplimiento del Plan de Desarrollo Institucional 2005-2009 [8]. Este programa se fundamenta en las políticas del Programa Institucional de Tutorías (PIT) 2002 [9], de nuestra institución; y su desarrollo conduciría a establecerlo de manera permanente para la resolución de la problemática existente.

Mediante este programa se ofreció al estudiante el apoyo y supervisión pertinentes en temas que representen mayor dificultad con el fin de abatir las deficiencias y rezagos académicos y cumplir con los objetivos de enseñanza aprendizaje planteados en cada una de las asignaturas contenidas en el plan de estudios de las licenciaturas de Químico Biólogo Clínico y Químico en Alimentos.

El procedimiento que se siguió en el desarrollo del programa de asesorías académicas para los alumnos del área básica del Departamento de Ciencias Químico Biológicas fue el siguiente:

1. Se analizaron las estadísticas de alumnos inscritos, aprobados, reprobados y el porcentaje de aprobación en las materias de Química del área básica: Química General, Química Inorgánica, Química Orgánica I, II y III, Química Analítica I y II, Bioquímica I y II, Termodinámica Química, Equilibrio Químico y Cinética Química, de los semestres 2004-1 hasta el 2007-2 (Tabla 1 y Gráfica 1). (Información obtenida de la Dirección de Servicios Escolares de la Universidad de Sonora).

En éstas se observa una disminución en el porcentaje de aprobación. Por ejemplo en el semestre 2004-2 se obtuvieron porcentajes de aprobación superiores al 60 en todas las materias, pero en semestres posteriores se obtuvieron porcentajes inferiores al 60 . Tal es el caso de los semestres 2006-2 y 2007-2, en los cuales de las 12 materias del área básica, 7 y 4 materias en los semestres respectivos, registraron una disminución del porcentaje de aprobación a menos del 60.

Tabla 1. Situación del Departamento de Ciencias Químico Biológicas en los ciclos anteriores al programa de asesoría académica, indicada como porcentajes de aprobación en las diferentes asignaturas de Química del Área Básica, obtenidos en los períodos del 2004-1 al 2007-2

\begin{tabular}{|c|c|c|c|c|c|c|c|c|}
\hline Materia & 2004-1 & 2004-2 & 2005-1 & 2005-2 & 2006-1 & 2006-2 & 2007-1 & 2007-2 \\
\hline Q. General & 78.6 & 69.3 & 55.3 & 62.8 & 65.4 & 63.1 & 47.6 & 63.1 \\
\hline Q. Inorgánica & 70.5 & 70.2 & 53.3 & 64.7 & 71 & 42.5 & 83.5 & 55.5 \\
\hline Q. Orgánica I & 69.6 & 90.2 & 88 & 71 & 79.1 & 21.4 & 69.5 & 46.9 \\
\hline Q. Orgánica II & 71.9 & 82.4 & 76.3 & 53.8 & 71.7 & 56.8 & 59 & 61.5 \\
\hline Q. Orgánica III & 57.9 & 77.3 & 89 & 79.2 & 86.4 & 46.3 & & 58.8 \\
\hline Termodinámica & 83.3 & 82.9 & 72.4 & 68 & 73.6 & 66 & 67.7 & 63.8 \\
\hline Equilibrio Q. & 55.5 & 75.4 & 81.2 & 59 & 70.8 & 47.4 & 74.5 & 61.2 \\
\hline Cinética Q. & 72 & 80.7 & 78.9 & 88.5 & 77.8 & 83.3 & 82.8 & 72.6 \\
\hline Q. Analítica I & 73 & 70.5 & 75 & 64.7 & 82.7 & 57 & 80 & 75.8 \\
\hline Q. Analítica II & 71.4 & 65.8 & 96.7 & 84.6 & 51.3 & 56.5 & 77.4 & 55.7 \\
\hline Bioquímica I & 82.2 & 84.9 & 82.3 & 72.6 & 57.1 & 61.3 & 76.9 & 60.8 \\
\hline Bioquímica II & 58.8 & 98.9 & 67.9 & 93.3 & 84.2 & 79.2 & 89.7 & 84.3 \\
\hline Promedios & 70.39 & 79.04 & 76.35 & 71.85 & 72.59 & 56.7 & 73.51 & 63.3 \\
\hline
\end{tabular}




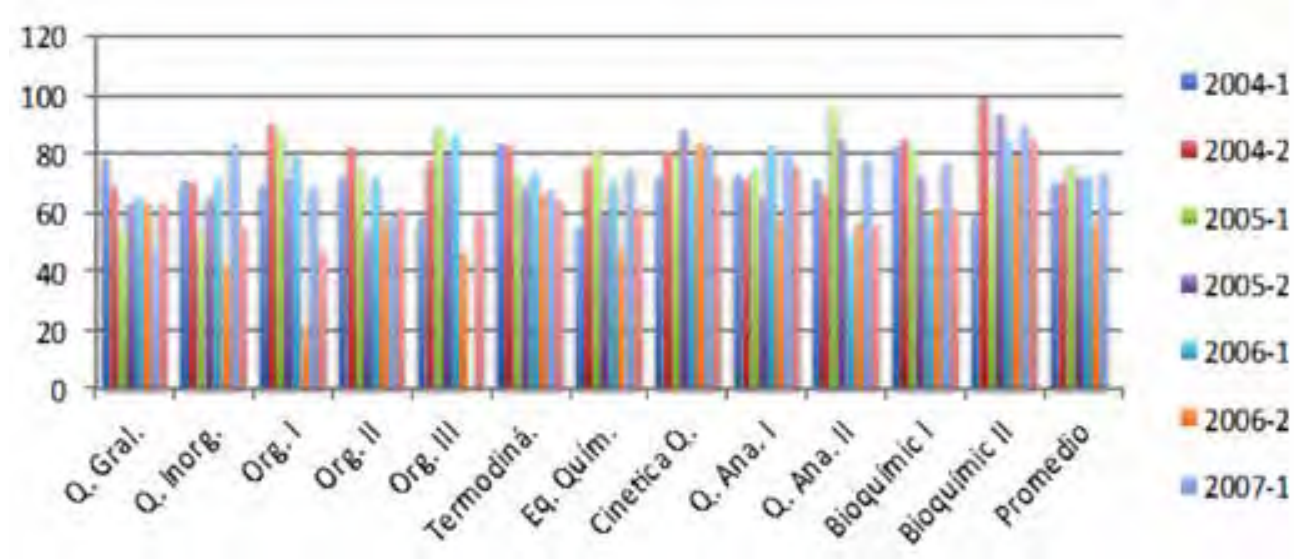

Gráfica 1. Situación del Departamento de Ciencias Químico Biológicas en los ciclos anteriores a la asesoría académica. Porcentajes de aprobación en las diferentes asignaturas de Química del área básica obtenidos en los períodos del 2004-1 al 2007-1.

2. Con el fin de establecer objetivamente la necesidad del proyecto de asesorías, se realizó una encuesta a los alumnos de área básica mediante un cuestionario de diez preguntas sobre atención del profesor a las necesidades de asesoría para abatir el índice de reprobación. Así mismo se consensó la aceptación de un programa de asesorías extra clase, con preguntas sobre su disponibilidad para aprovechar dicho proyecto y alternativas de organización.

En esta encuesta, para evaluar la intervención del maestro para abatir el índice de reprobación, el $85 \%$ de los estudiantes encuestados señala la falta de retroalimentación de la clase, el $86.8 \%$ señala que los maestros manifiestan no tener suficiente tiempo para ofrecer asesorías extractase y $80 \%$ señala que las clases no son suficientemente claras. En lo que se refiere a preguntas sobre el cumplimiento del programa y la puntualidad y asistencia del maestro y aspectos de evaluación, el $91 \%$ de los alumnos encuestados, evaluaron muy bien al profesorado.

3. Con los resultados obtenidos anteriormente, se decidió iniciar las asesorías académicas con los alumnos que se encontraban en riesgo, o sea aquellos que cursaran las materias de Química del área básica por segunda o tercera vez. Para identificar a la población en riesgo se solicitó a la Dirección de Servicios Escolares de la Universidad de Sonora las listas de los alumnos que cursaban por segunda y tercera vez alguna de las materias ya mencionadas, junto con sus horarios de clase [10].

4. Con base en los horarios de los alumnos en riesgo se programaron asesorías en los espacios de tiempo disponibles para dichos alumnos. Los maestros de tiempo completo, adecuaron su tiempo al horario disponible de los alumnos; para impartir la asesoría. Cabe destacar, que la participación del maestro fue voluntaria y además colaboraron dos estudiantes por semestre, a los que se les apoyó con beca ayudantía, proporcionada a alumnos que cumplieran con los requisitos establecidos en convocatoria emitida por la División de Ciencias Biológicas y de la Salud.

5. Los horarios disponibles para el programa de asesorías, fueron publicados en todos los edificios del Departamento, además se entregó un tríptico conteniendo toda la información del programa, para los alumnos que requerían de ésta y una carta personalizada del Jefe de Departamento invitándolos a participar, comentando del beneficio o bondades que ofrecía el programa.

6. Al implementar el programa de asesorías, se llevó un registro de asistencias y temas tratados

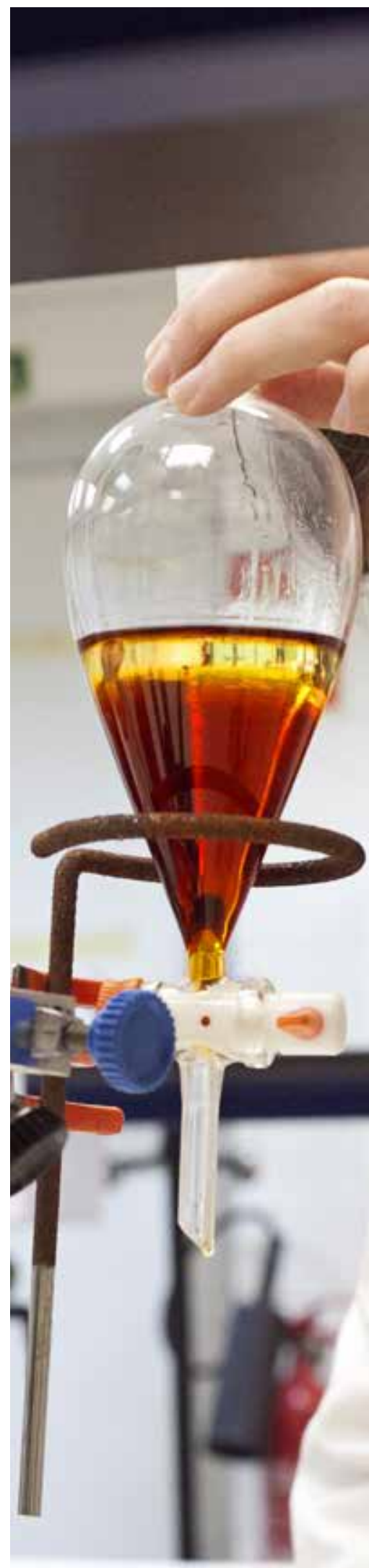

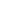


con cada alumno o grupo de alumnos asesorados firmado por el (los) alumno(s) y el maestro asesor (Se sugirió que la primera asesoría fuera individual para poder conocer la causa de la irregularidad del alumno ya fuesen problemas personales o de aprendizaje). Las asesorías, grupales e individuales (si fuera necesario), deberían ser semanales y tener una duración hasta de una hora.

7. Las asesorías proporcionaron al estudiante el apoyo requerido en los temas de mayor dificultad en las diferentes asignaturas utilizando:

- Reafirmación temática

- Resolución de dudas

- Resolución de ejercicios

- Aplicación de casos prácticos

- Intercambios de experiencias

8. Se reportó, al responsable del programa, todas las asesorías brindadas, en un formato de registro que contenía: nombre del alumno, número de expediente, fecha y número de sesiones, los temas tratados en cada una de éstas y el resultado final obtenido (aprobado o reprobado).

El programa de asesorías fue implementado a partir del semestre 2008-2. Tuvo una población inicial de 197 alumnos en riesgo, de los cuales 58 alumnos $(29,4 \%)$ respondieron al programa, aprobando los cursos del semestre correspondiente, 44 alumnos (75,8 \%). En el semestre 2009-1, se registró un total de 239 alumnos en riesgo, asistiendo únicamente $30(12.5 \%)$ a asesoría de los cuales 22 aprobaron los cursos del semestre (73.3 $\%)$. Es importante señalar que en este último semestre se paralizaron las actividades de la Universidad por un período de tiempo debido al estallamiento de huelga; a pesar de esta situación, el programa de asesorías pudo reestablecerse y obtener el porcentaje reportado anteriormente, lo cual indica el buen funcionamiento del programa (tabla 2).

Tabla 2. Total de alumnos en riesgo reprobados en una o más materias del área básica, asistentes a la asesoría y los que se recuperaron en los semestres 2008-2 y 2009-1

\begin{tabular}{|c|c|c|c|c|}
\hline Semestre & $\begin{array}{c}\text { Número de } \\
\text { alumnos en } \\
\text { riesgo }\end{array}$ & $\begin{array}{c}\text { Número de } \\
\text { alumnos } \\
\text { asesorados }\end{array}$ & $\begin{array}{c}\text { Número de } \\
\text { alumnos } \\
\text { asesorados } \\
\mathbf{y} \\
\text { aprobados }\end{array}$ & $\begin{array}{c}\text { Porcentaje } \\
\text { de } \\
\text { aprobados }\end{array}$ \\
\hline $\mathbf{2 0 0 8 - 2}$ & 197 & 58 & 44 & 75.8 \\
\hline $\mathbf{2 0 0 9 - 1}$ & 239 & 30 & 22 & 73.3 \\
\hline
\end{tabular}

Finalmente, se analizaron los resultados de las actas de calificaciones en las materias de Química del área básica del semestre 2008-2 y 2009-1 (ver tablas 3,4 y 5). Se

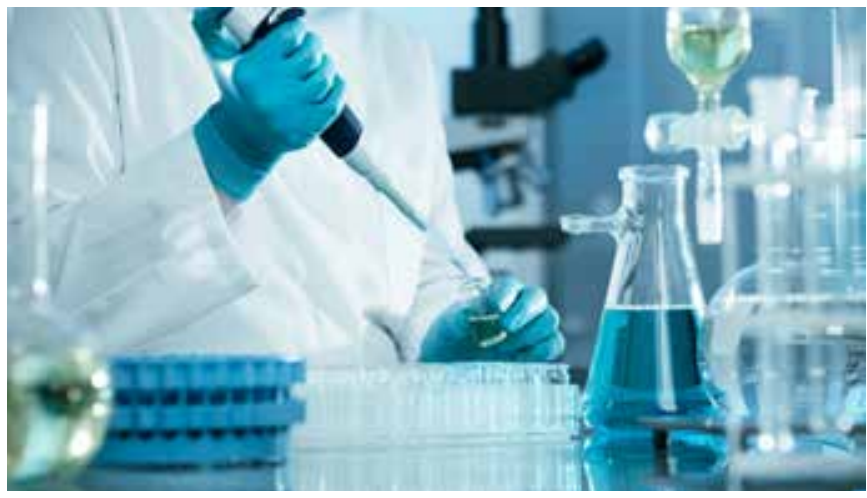

observa que hay materias cuyo porcentaje de aprobación no aumentó luego de haber implementado el programa de asesorías, permaneciendo en torno al $60 \%$, lo que indica que tales materias (Química General, Química Orgánica, Termodinámica Química y Bioquímica) deben recibir un trato intensivo, para poder mejorar el índice de aprobación. Los resultados obtenidos en los ciclos 2008-2 y 2009-1 (tabla 3, 4 y 5), muestran 550 alumnos aprobados (66.34 \%) en el 2008-1 y 663 (69.71\%) para el 2009-1.

Comparando con los porcentajes de aprobación obtenidos en el primer año de la asesoría: 2008-2 (75.8\%) y 2009-1 (73.3\%) representaron una recuperación del 8.00 $\%$ de los alumnos en riesgo del ciclo 2008-2, disminuyendo el índice de reprobación en $13.62 \%$ y un 3.31\% en el ciclo 2009-1 disminuyendo el índice de reprobación en $7.09 \%$ (tablas 2 y 5 ).

Tabla 3. Resultados obtenidos en las actas de calificaciones semestrales ciclo 2008-2 en las materias de química del área básica.

\begin{tabular}{|l|c|c|c|c|}
\hline \multicolumn{1}{|c|}{ Materia } & $\begin{array}{c}\text { Alumnos } \\
\text { inscritos }\end{array}$ & $\begin{array}{c}\text { Alumnos } \\
\text { aprobados }\end{array}$ & $\begin{array}{c}\text { Alumnos } \\
\text { reprobados }\end{array}$ & $\begin{array}{c}\text { Porcentaje } \\
\text { de } \\
\text { aprobación }\end{array}$ \\
\hline Q. General & 44 & 21 & 23 & 47.7 \\
\hline Q. Inorgánica & 33 & 21 & 12 & 63.3 \\
\hline Q. Orgánica I & 36 & 17 & 19 & 47.2 \\
\hline Q. Orgánica II & 134 & 73 & 61 & 54.5 \\
\hline Q. Orgánica III & 52 & 38 & 14 & 73.1 \\
\hline Termodinámica & 61 & 35 & 26 & 57.3 \\
\hline Equilibrio Q. & 43 & 30 & 13 & 60.8 \\
\hline Cinética Q. & 63 & 50 & 13 & 79.4 \\
\hline Q. Analítica I & 137 & 105 & 32 & 76.6 \\
\hline Q. Analítica II & 46 & 41 & 5 & 89.1 \\
\hline Bioquímica I & 138 & 82 & 56 & 59.4 \\
\hline Bioquímica II & 42 & 37 & 5 & 88.1 \\
\hline Total & 829 & 550 & 279 & 66.34 \\
\hline
\end{tabular}


Tabla 4. Resultados obtenidos en las actas de calificaciones semestrales ciclo 2009-1 en las materias de química del área básica.

\begin{tabular}{|l|c|c|c|c|}
\hline Materia & $\begin{array}{c}\text { Alumnos } \\
\text { inscritos }\end{array}$ & $\begin{array}{c}\text { Alumnos } \\
\text { aprobados }\end{array}$ & $\begin{array}{c}\text { Alumnos } \\
\text { reprobados }\end{array}$ & $\begin{array}{c}\text { Porcentaje } \\
\text { de } \\
\text { aprobación }\end{array}$ \\
\hline Q. General & 53 & 37 & 16 & 69.8 \\
\hline Q. Inorgánica & 164 & 117 & 47 & 71.3 \\
\hline Q. Orgánica I & 161 & 116 & 45 & 72 \\
\hline Q. Orgánica II & 59 & 53 & 6 & 89.8 \\
\hline Q. Orgánica III & 91 & 54 & 37 & 59.3 \\
\hline Termodinámica & 61 & 29 & 32 & 47.5 \\
\hline Equilibrio Q. & 89 & 63 & 26 & 70.7 \\
\hline Cinética Q. & 46 & 38 & 8 & 82.6 \\
\hline Q. Analítica I & 42 & 33 & 9 & 78.5 \\
\hline Q. Analíticall & 59 & 42 & 17 & 71.1 \\
\hline Bioquímica I & 68 & 33 & 35 & 48.5 \\
\hline Bioquímica II & 58 & 48 & 10 & 82.7 \\
\hline Total & 951 & 663 & 288 & 69.7 \\
\hline
\end{tabular}

Tabla 5. Resultados obtenidos en las actas de calificaciones en las carreras de QBC y QA en las materias de Química del Área Básica en los ciclos 2008-2 y 2009-1

\begin{tabular}{|l|l|l|l|l|} 
Semestre & $\begin{array}{c}\text { Número de } \\
\text { alumnos } \\
\text { inscritos }\end{array}$ & $\begin{array}{c}\text { Número de } \\
\text { alumnos } \\
\text { aprobados }\end{array}$ & $\begin{array}{c}\text { Número de } \\
\text { alumnos } \\
\text { reprobados }\end{array}$ & $\begin{array}{c}\text { Porcentaje } \\
\text { de } \\
\text { aprobados }\end{array}$ \\
\hline $2008-2$ & 829 & 550 & 279 & 66.34 \\
\hline $2009-1$ & 951 & 663 & 288 & 69.71 \\
\hline
\end{tabular}

Actualmente, semestre 2016-2, se cumplen 8 años de implementación del programa, el cual ha tenido algunos cambios, entre ellos, que la asesoría se ofrece a todos los alumnos, tanto regulares como irregulares o en riesgo y, el más importante, que las asesorías del programa ya no las realizan los maestros de tiempo completo sino que son atendidas al $100 \%$ por alumnos destacados de semestres avanzados (asesorías de pares), esto ayuda a la dinámica educativa actual, la cual demanda un sinnúmero de actividades extra-clase del profesorado, que le obliga prácticamente a desatender al estudiante $\mathrm{o}$ a reducir la asesoría a una sesión de clase antes de realizar los exámenes parciales [11].

\section{CONCLUSIÓN}

La implementación del Programa de Asesorías, logró recuperar un $29.44 \%$ de los alumnos que ingresaron al programa en el ciclo $2008-1$ y $12.55 \%$ en el 2009 -
1, disminuyendo el índice de reprobación del total de alumnos que si asistieron a asesorías.

Actualmente se destaca la continuidad del programa tras 8 años de sus inicios, con "asesoría de pares" llevada a cabo por alumnos sobresalientes de semestres avanzados, que por un lado favorecen su formación académica al desarrollar habilidades para enseñar y por otro lado, proporcionan confianza a los alumnos asesorados que son los participantes activos y que reciben atención personalizada con la asesoría de los propios estudiantes, fortaleciendo así sus habilidades de estudio y trabajo. Sin embargo, aún no es posible determinar la efectividad total del programa de asesorías, ya que se requieren datos de seguimiento de estudiantes con rezago que no participaron en el programa.

\section{BIBLIOGRAFÍA}

[1] R.M.E. Chávez Rocha, C.R. Vargas Cortez. (2007) "El papel de la asesoría académica en los programas de tutorías: caso itt", Tiempo de educar, vol 8(15).

[2] A. López Suárez, A. Albíter Rodríguez, L. Ramírez Revueltas. (2008) "Eficiencia terminal en la educación superior, la necesidad de un nuevo paradigma", Revista de la Educación Superior Vol.37 (2), No. 146, pp. 135-151.

[3] A.L. Zarza García. R. C. Lara Severino, R. D. Iznaga Ravelo, L. Maas Góngora. (2012) Universidad Autónoma del Carmen. Accessed sept 2016. [Online]. http://www.unacar.mx/ contenido/difusion/acalan65pdf/contenido.pdf

[4] (2011) Programa de asesorías académicas, Instituto de Estudios Superiores de Chiapas. Accessed sept 2016 [Online]. http://www.iesch.edu.mx/tuxtla/wp-content/ uploads/2011/08/Programa-de-Asesorias-Academicas.pdf

[5] (2015) Programa de asesoría académica, Universidad Autónoma Chapingo. Accessed sept 2016 [Online]. http:// dima.chapingo.mx/contenido/reglamentos/Programa_de_ asesoria_academica.pdf

[6] Izar, J.; Ynzunza, C.; López, H. (2011). Factores que afectan el desempeño académico de los estudiantes de nivel superior en Rioverde, San Luis Potosí, México. CPU-e, Revista de Investigación Educativa, 12. Consultado septiembre 2016 en: http://www.uv.mx/cpue/ num12/opinion/lzar-desempeño academico.html

[7] M.A. Pacheco, B. Burgos. (2007) "Estudio de deserción en la Universidad de Sonora", Colección Documentos de Investigación Educativa. México.

[8] Plan de Desarrollo Institucional 2005-2009. Universidad de Sonora, México.

[9] Programa Institucional de Tutorías (PIT) 2002. Universidad de Sonora, México

[10] H.P. Aguilar, y J.I.P. Aguilar. (1999) Plan para incrementar los índices de acreditación y reducir la deserción en las escuelas de ingeniería. Memorias del Primer Foro de Enseñanza de las Matemáticas. Facultad de Ingeniería, UNAM, México.

[11]Álvarez, P. y col. (2005). La tutoría entre iguales y la orientación universitaria: una experiencia de formación académica y profesional. Revista Educar [en línea], 36. Recuperado el 30 de julio de 2009 de: http://dialnet.unirioja. es/servlet/articulo?codigo $=1399494$. 